\title{
Evidences of unconventional cosmic ray propagation in gamma-ray and neutrino astronomy
}

\section{Gaggero}

GRAPPA, University of Amsterdam, Science Park, 1090 GL Amsterdam, The Netherlands

\section{Grasso*, A. Marinelli}

I.N.F.N. and Dipartimento di Fisica, Università di Pisa, Largo Pontecorvo, 3, I-56127 Pisa, Italy E-mail: dario.grasso@pi.infn.it

\section{Taoso}

Instituto de Fisica Teorica UAM/CSIC, calle Cabrera 13, 28049 Cantoblanco, Madrid, Spain

\section{Urbano}

CERN, Theory division, CH-1211 Genève 23, Switzerland

\section{Valli}

I.N.F.N. and Dipartimento di Fisica, Università di Roma "la Sapienza", Largo A. Moro, Roma, Italy

\begin{abstract}
Several independent analyses of Fermi-LAT results found evidences of a spatial dependence of the cosmic ray $(\mathrm{CR})$ proton spectral index which is not accounted for conventional models of CR transport in the Galaxy. We show that these results may have a relevant impact on the gamma-ray and neutrino diffuse emissions of the Galaxy above the TeV. Indeed a phenomenological model which adopts a spatial dependent diffusion coefficient, so to account for those features, also reproduces the gamma-ray excess, at $15 \mathrm{TeV}$, found by Milagro and H.E.S.S. measurements in the inner Galactic plane. The same model predicts a neutrino emission from that region which is significantly larger than expected on the basis of conventional models. This emission is compatible with ANTARES and IceCube results and it is a natural target for KM3NeT.
\end{abstract}

Neutrino Oscillation Workshop

4 - 11 September, 2016

Otranto (Lecce, Italy)

\footnotetext{
* Speaker.
} 


\section{Introduction}

The IceCube collaboration recently released updated measurements of the spectrum of the astrophysical high-energy neutrino flux based on high-energy starting events (HESE) [1] and on passing muons ones [2]. Interestingly, the results of those analysis are significantly different, the former finding a higher and softer spectrum. Due to the geographical position of the IceCube observatory, HESE events are predominantly coming form the Southern hemisphere, where the inner region of the Galaxy is located, while passing muons events come only from the Northern one. Therefore, several authors suggested that the aforementioned discrepancy may be due to the presence of a significant Galactic components in Southern sky (see e.g. [3] and Ref.s therein). A similar conclusion was also reached on the basis of claims of the presence of an excess of IceCube events along the Galactic Plane (GP) which, however, are still rather controversial.

Here we investigate this issue following a different approach: we model the diffuse neutrino emission of the Galaxy on the basis of most updated cosmic rays (CR) and $\gamma$-ray data sets. Although this exercise was done already several times, those computations were based on conventional $\mathrm{CR}$ transport models which assume space independent diffusion. Recently, however, this assumption was questioned by the results of several independent analyses of Fermi-LAT $\gamma$-ray data which found evidences of a continuos hardening of the primary CR spectrum getting closer to the GC along the Galactic plane (GP). In the absence of any evidence of a spatially dependent source spectrum, that behaviour can only be interpreted in terms of inhomogeneous $\mathrm{CR}$ transport.

Below we will show that a model which was developed to reproduce Fermi-LAT data in those terms solves well know anomalies faced by conventional models. Indeed that model reproduces Milagro and H.E.S.S. measurements of the diffuse $\gamma$-ray emission above the $\mathrm{TeV}$ in the inner Galactic plane. We will then use the same model to compute the diffuse neutrino emission in the same region an compare our predictions with ANTARES and IceCube results.

\section{A new CR propagation model against high-energy $\gamma$-ray data}

The model proposed in [4] $\left(\mathrm{KRA}_{\gamma}\right)$ assumes that the exponent $\delta$ setting the rigidity dependence of the CR diffusion coefficient has the following Galactocentric radial dependence: $\delta(R)=A R+B$, where $A=0.035 \mathrm{kpc}^{-1}$ and $B=0.21$ so that $\delta\left(R_{\odot}\right)=0.5$. That setup was implemented wih DRAGON, a numerical code designed to compute the propagation of all CR species [5] in the general framework of position-dependent diffusion. Only proton and Helium CR nuclei need to be considered here since heavier species give a negligible contribution to the $\gamma$-ray and neutrino emissions. For their primary spectra here we assume a broken power law with index $\Gamma=2.35 / 2.48$ below/above $\sim 250 \mathrm{GeV} / \mathrm{n}$ and an exponential cutoff at $E_{\text {cut }}=50 \mathrm{PeV}$ such to reproduce PAMELA, CREAM and KASCADE-Grande data. The $\mathrm{KRA}_{\gamma}$ model was build to reproduce the Galactic diffuse $\gamma$-ray emission measured by Fermi-LAT both at low and at mid Galactic latitudes without spoiling local CR observables. Noticeably this setup does better than conventional models especially at high energies $(E \gtrsim 10 \mathrm{GeV}$ ). Therefore, Milagro and H.E.S.S. observatories, which measured the diffuse $\gamma$-ray emission in the inner GP above the TeV, provide a valuable test of that model. 

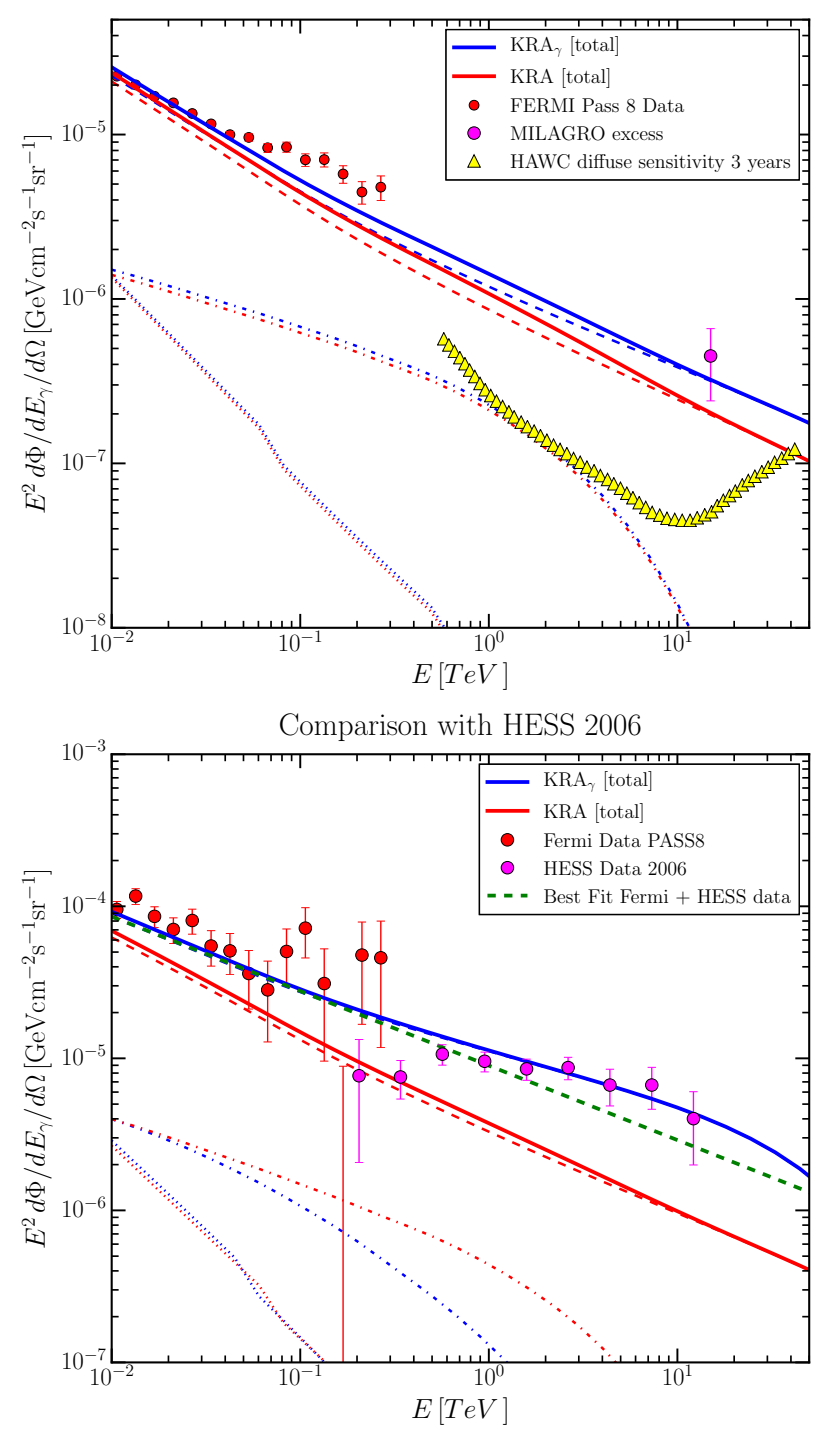

Figure 1: Upper panel: The diffuse emission $\gamma$-ray spectrum measured by Fermi-LAT (PASS8) and Milagro in the inner Galactic plane $\left(|b|<2^{\circ}, 30^{\circ}<l<65^{\circ}\right)$ is compared with the KRA (conventional) and KRA $\gamma$ model predictions. The expected sensitivity of HAWC is reported. The main spectral components $\left(\pi^{0}\right.$ decay: dashed; Inverse Compton: dot-dashed) are also shown. Lower panel: The same models are compared with H.E.S.S. and Fermi-LAT (PASS8) data for the diffuse emission in the Galactic ridge region: $|l|<0.8^{\circ}$, $|b|<0.3^{\circ}$. Point sources from the 3FGL Fermi catalogue are subtracted from those data.

Milagro water Cherenkov observatory measured the $\gamma$-ray flux in the sky window with $|b|<2^{\circ}$ and $30^{\circ}<l<65^{\circ}$ at a median energy of $15 \mathrm{TeV}$. This was found to be $4 \sigma$ above the predictions of a conventional models tuned on CR data available in 2008 [6]. The Milagro anomaly holds even considering updated conventional models based on Femi-LAT data. This is visible in Fig. 1 (left panel) where a reference conventional (KRA) model, tuned to reproduce local CR data as well as the large scale $\gamma$-ray diffuse emission measured by Fermi-LAT, falls short from Milagro data point of several sigmas. From Fig. 1 the readers can see as instead the KRA $\gamma$ model matches the Milagro result. See Ref.[7] for more details. 


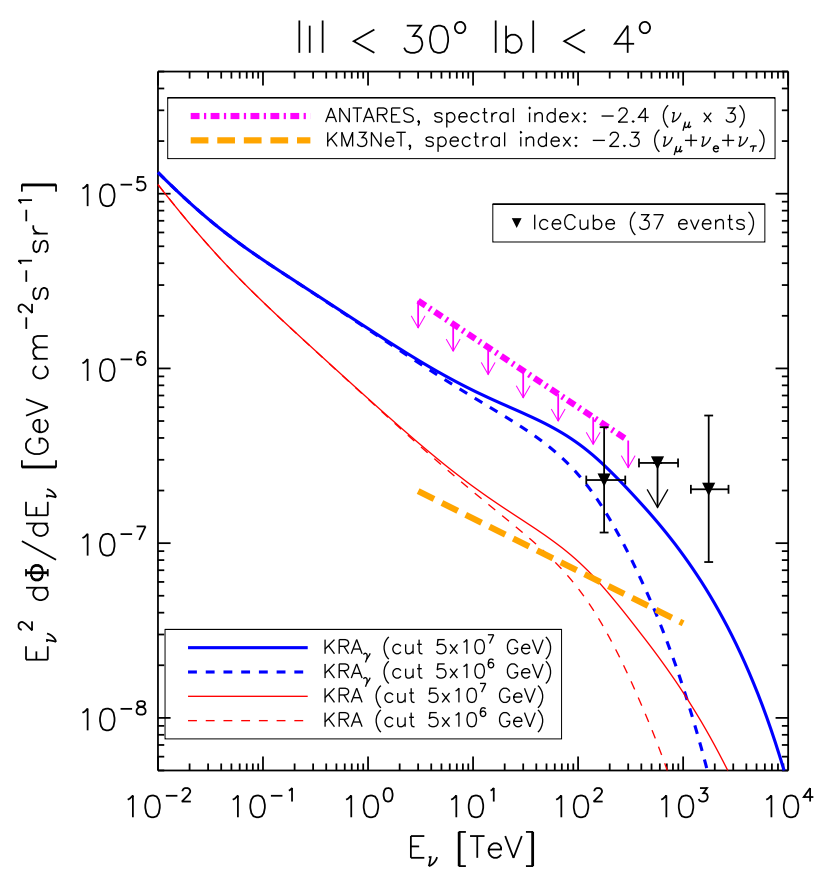

Figure 2: Total neutrino spectra in the inner Galactic plane computed for the conventional KRA and the novel $\mathrm{KRA}_{\gamma}$ models for two different cutoff of CR primaries. We also show the maximal flux, estimated considering 3 years of IceCube HESE events, the constraint from ANTARES experiment as well as the deduced sensitivity of the future Mediterranean observatory KM3NeT after 4 years of lifetime.

We also compare the KRA $\gamma$ model with H.E.S.S. [8] and Fermi-LAT data in the Galactic ridge region: $|l|<0.8^{\circ},|b|<0.3^{\circ}$. As shown in Fig. 1 (right panel) a single power law with index $\sim 2.5$ - implying a $\Gamma_{C R} \sim 2.4$ for $\mathrm{CR}$ due to the weak energy dependence of the $p p$ scattering cross section - provides a satisfactory fit of those data. This is significantly harder than expected for conventional models. Again, the $\mathrm{KRA}_{\gamma}$ model is in much better agreement with the data.

\section{Neutrino diffuse emission of the Galaxy}

Corroborated by these positive results we pass to compute the expected diffuse neutrino emission of the Galaxy.

Here we consider the sky window $|l|<30^{\circ}$ and $|b|<4^{\circ}$ where the Galactic neutrino emission is expected to provide the dominant contribution to the observed flux. For this region the ANTARES collaboration provided an upper limit on the muon neutrino flux based on events collected between 2007 and 2013 in the energy range [3 $\div 300$ ] TeV [9]. In Fig. 2 we compare the neutrino flux computed with the KRA (conventional model) and $\mathrm{KRA}_{\gamma}$ setups with that experimental constraint. We notice the large enhancement (almost a factor of 5 at $100 \mathrm{TeV}$ ) obtained with the $\mathrm{KRA}_{\gamma}$ model respect to the conventional scenario. Indeed, while we find that the flux corresponding to the KRA model may require long time of observation even by the KM3NeT observatory, our prediction for the $\mathrm{KRA}_{\gamma}$ model is instead well above the sensitivity reachable by that experiment 
in 4 years and it is almost within the ANTARES observation capabilities (see also [10] where a similar result was reached with an analytical approach).

A good agreement with IceCube results was also found on the whole Galactic plane (see e.g. Fig. 1 in [11]). On the whole sky, the diffuse Galactic emission computed with the KRA $\gamma$ model can account up to $\sim 15 \%$ (to be compared to $\sim 8 \%$ obtained for the conventional set-up) of the flux measured by IceCube. This is pretty compatible with present upper limits based on the angular distribution of events and could be detectable by forthcoming IceCube analyses.

\section{References}

[1] M. G. Aartsen et al. [IceCube Collaboration], Phys. Rev. Lett. 113 (2014) 101101; C. Kopper et al. [IceCube Collaboration], PoS ICRC 2015 (2016) 1081.

[2] M. G. Aartsen et al. [IceCube Collaboration], Astrophys. J. 833 (2016) no.1, 3.

[3] A. Palladino, M. Spurio and F. Vissani, JCAP 1612 (2016) no.12, 045.

[4] D. Gaggero, A. Urbano, M. Valli and P. Ullio, Phys. Rev. D 91 (2015) no.8, 083012.

[5] C. Evoli, D. Gaggero, D. Grasso and L. Maccione, JCAP 0810 (2008) 018.

[6] A. A. Abdo et al., Astrophys. J. 688 (2008) 1078.

[7] D. Gaggero, D. Grasso, A. Marinelli, A. Urbano and M. Valli, Astrophys. J. 815 (2015) no.2, L25.

[8] F. Aharonian et al. [H.E.S.S. Collaboration], Nature 439 (2006) 695.

[9] S. Adrian-Martinez et al. [ANTARES Collaboration], Phys. Lett. B 760 (2016) 143.

[10] G. Pagliaroli, C. Evoli and F. L. Villante, JCAP 1611 (2016) no.11, 004.

[11] A. Marinelli, D. Gaggero, D. Grasso, A. Urbano and M. Valli, EPJ Web Conf. 116 (2016) 04009. 